\section{Recovery of Salmonella enterica Newport Introduced through Irrigation Water from Tomato (Lycopersicum esculentum) Fruit, Roots, Stems, and Leaves}

\author{
Leslie D. Hintz, Renee R. Boyer ${ }^{1}$, Monica A. Ponder, \\ and Robert C. Williams \\ Department of Food Science and Technology, Virginia Polytechnic Institute \\ and State University, 22 Duck Pond Drive, Blacksburg, VA 24061
}

\section{Steven L. Rideout \\ Virginia Tech Eastern Shore Agricultural Research and Extension Center, Virginia Polytechnic Institute and State University, 33446 Research Drive, Painter, VA 23420 \\ Additional index words. foodborne illness, outbreak, salmonellosis, Newport, irrigation, leaf, stem, roots, environmental growth chamber}

\begin{abstract}
Tomatoes have been associated with numerous outbreaks of salmonellosis in recent years. Trace-backs suggest tomato fruits may become contaminated during preharvest. The objective of this study was to determine the potential for Salmonella enterica serotype Newport to be internalized into the roots, stems, leaves, and fruit of red round tomato plants through contaminated irrigation water at various stages of plant development. Tomato plants were irrigated with 250 or $350 \mathrm{~mL}$ (depending on growth stage) of $7 \log \mathrm{CFU} \cdot \mathrm{mL}^{-1} S$. Newport-contaminated irrigation water every 7 days. Roots, stems, leaves, and two tomato fruit from plants irrigated with $S$. Newport or water (negative control) were sampled for contamination at five stages of growth. Twenty-five of the 92 total samples taken from plants irrigated with $S$. Newport were confirmed positive (serovar specificity was not evaluated). Sixty-five percent of confirmed samples were roots, $40 \%$ were stems, $10 \%$ were leaves, and $6 \%$ were fruit. There was a significant difference in the presence of $S$. enterica according to tissue sampled (roots $>$ stems $>$ leaves, and fruit) $(P<0.05)$ and no association between growth stage and contamination $(P>0.05)$. Contamination of tomato fruit with $S$. Newport introduced through irrigation water is low because a high level of persistent contamination of a plant in the agricultural setting is unlikely.
\end{abstract}

The presence of human pathogens on fresh produce is a significant public health concern because these products are often consumed raw. Since 1998, 13 outbreaks of Salmonella enterica related to fresh tomato consumption have occurred in the United States (J. Guzewich, personal communication). This large number of outbreaks prompted the U.S. Food and Drug Administration (FDA) to implement a multiyear initiative in 2007 aimed at reducing tomato-related illnesses (FDA, 2007a, 2007b). Goals of this initiative include identifying production practices or conditions that may lead to tomato contamination as well as facilitating and promoting research on tomato safety (FDA,

\footnotetext{
Received for publication 17 Dec. 2009. Accepted for publication 22 Jan. 2010.

We thank Dr. Hengjian Wang from the Virginia Tech Food Science and Technology Department for statistical consulting services.

${ }^{1}$ To whom reprint requests should be addressed; e-mail rrboyer@vt.edu.
}

2007a, 2007b). These outbreaks have specifically raised concerns about the potential for $S$. enterica to contaminate tomatoes in the preharvest environment.

In 2008, the value of fresh market tomato production in the United States was \$1.4 billion (National Agricultural Statistics Service, 2009). The impact of tomato-related foodborne outbreaks has significantly affected the tomato industry. A salmonellosis outbreak in 2008 was initially linked to tomatoes but later found to have a stronger association with jalapeno peppers (Centers for Disease Control and Prevention, 2008). This initial association between the outbreak and tomatoes is speculated to have cost the industry over $\$ 100$ million in losses (Shin, 2008). Because fresh market tomatoes are intended to be consumed raw, there are no steps during processing to eliminate the pathogen in the event that contamination occurred. It is essential for researchers to identify contamination routes to prevent outbreaks and further damage to the industry. If routes were identified, recommendations for steps that growers can take in the preharvest environment can be made to help reduce the risk of contamination.

Several potential pathways for $S$. enterica to contaminate tomato fruits have been examined by researchers. Tomato flowers brushed with a five-strain cocktail of $S$. enterica (serovars Enteritidis, Hartford, Michigan, Montevideo, and Poona) resulted in $25 \%$ of ripened fruit contaminated (Guo et al., 2001). Additionally, plants inoculated by stem injection $5 \mathrm{~cm}$ below the flower base also produced contaminated fruit (Guo et al., 2001). Irrigation of plants with contaminated water may also be a potential source of fruit contamination. Internalization of $S$. enterica into the hypocotyls and cotyledons, stems, and leaves of tomato seedlings occurred after 1, 5, and $9 \mathrm{~d}$ of growth when grown hydroponically in contaminated nutrient solution (Guo et al., 2002). However, when $S$. Enteritidiscontaminated water was applied directly onto the soil of pots containing the tomato cultivar Cherry Gold, the pathogen was not recovered from plant tissue (Jablasone et al., 2004). Greenhouse tomato plants irrigated with $S$. Montevideo-contaminated water displayed no contamination in tomato fruits, but samples of roots exposed to four or more irrigation events (once every $14 \mathrm{~d}$ ) were positive (Miles et al., 2009).

The primary $S$. enterica serovars involved in tomato outbreaks are Newport, Javiana, and Braenderup (J. Guzewich, personal communication). Studies tracing potential colonization by tomato outbreak strains through the root system into tomato plants from seedlings to fruit have not been conducted. The objective of this study was to determine whether tomato plants irrigated with $S$. Newport have the potential to uptake the organism through roots and have translocation throughout the plant resulting in contaminated tomato fruit.

\section{Materials and Methods}

Bacterial culture. A clinical isolate of $S$. Newport (J1893) was obtained from the Centers for Disease Control and Prevention (Atlanta, GA) for use in this study. The isolate originated from a human case of salmonellosis associated with the 2005 tomato outbreak. On receipt, the isolate was plated for isolation and confirmation onto XLT-4 agar (Becton Dickson, Sparks, MD) and incubated for $24 \mathrm{~h}$ at $37^{\circ} \mathrm{C}$. Colonies presumptively positive for $S$. enterica were red with black centers indicating $\mathrm{H}_{2} \mathrm{~S}$ production. The isolates' serotype was confirmed using the Salmonella latex agglutination kit (Microbiology International, Frederick, MD) with antisera specific to group C2 (98\% specificity) to confirm $S$. Newport. After confirmation, the culture was stored at $-80{ }^{\circ} \mathrm{C}$ in $30 \%$ glycerol and tryptic soy broth (TSB; Becton Dickson) until use.

Inoculum preparation. To begin each experiment, cells were activated by three successive $24-\mathrm{h}$ incubations at $37^{\circ} \mathrm{C}$ in TSB. The final suspension was centrifuged at 7500 
$g$ for $10 \mathrm{~min}$ and the spent medium decanted. The culture was resuspended in sterile deionized (DI) water to create a final inoculum concentration of $7 \log \mathrm{CFU} \cdot \mathrm{mL}^{-1}$. Each set of the $S$. Newport suspension water was enumerated onto XLT-4 agar immediately before watering tomato plants to confirm inoculum concentration using surface plating. Plates were incubated at $37^{\circ} \mathrm{C}$ for $24 \mathrm{~h}$.

Tomato growth and irrigation parameters. Commercially produced 7-week-old tomato seedlings (cv. Solar Fire) were obtained from Virginia Tech's Eastern Shore Agricultural Research and Extension Center (ESAREC, Painter, VA) in a 128-cell Styrofoam plug tray (Speedling Incorporated, Sun City, FL) with Miracle Grow $®$ : Seed Starting Potting Mix (Scotts, Marysville, OH). Forty-four seedlings were planted in steam-sterilized sandy loam Bojac series soil obtained from the ESAREC in $15.6 \mathrm{~L}$ (3 gal.) sterilized round terra cotta pots. The 44 pots were evenly distributed between two Conviron CMP5090 (Winnipeg, Canada) reach-in controlled environmental chambers $\left(8^{\prime} \times 3^{\prime} \times 8^{\prime}\right)$ under a day and night cycle of $12 \mathrm{~h}$. One chamber contained the $S$. Newport-inoculated plants, and the other chamber contained the controls to prevent possible cross-contamination. For both chambers, the temperature was maintained at $28{ }^{\circ} \mathrm{C}$ (daytime) and $26{ }^{\circ} \mathrm{C}$ (nighttime) with relative humidity of $60 \%$. Once plants were $\cdot 3 \mathrm{ft}$ tall, sterilized stakes were inserted into the edge of the pots on either side of the plant and string secured the stems to ensure vertical growth and mirror commercial practices. In addition to irrigation (described subsequently), tomatoes received a Miracle Grow ${ }^{\circledR}$ : Tomato Plant Fertilizer (Scotts) treatment, per manufacturer's instructions, once a week, or as yellowing developed, throughout the growing period. The soil was kept moist but not saturated to prevent the soil from becoming anaerobic. Each pot was individually placed in a polypropylene tray to collect the irrigation runoff.

Plants were divided into two treatment groups: control (irrigated with distilled water) and experimental (irrigated with $S$. Newportcontaminated water at 7-d intervals). All plants, regardless of treatment group, received daily irrigation applied individually to the top of the soil. Care was taken to apply water in a constant pour directly at the base of the plant; no splash or contact between the treatment and the rest of the plant was made. The volume of water used to irrigate the tomato plants was constant between plants and dependent on the soil capacity and growth stage of the tomato plants. Initially, transplants were irrigated with $250 \mathrm{~mL}$ of solution until flowering when the irrigation aliquot was increased to $350 \mathrm{~mL}$ to account for additional water requirements. Plants in the experimental group were treated with a $7 \log \mathrm{CFU} \cdot \mathrm{mL}^{-1} S$. Newport suspension.

Tomato plant sampling. Tomato plants were randomly sampled for the presence of S. enterica (serovar specificity was not completed) at various tomato growth stages: $14 \mathrm{~d}$ posttransplant (PT), early fruit, midfruit, full fruit, and terminal. The early fruit sampling date was conducted when the plants had two or more green fruit $(49 \mathrm{~d}$ PT). The midfruit assessment date corresponded to when the plants contained a mixture of red and green fruit (56 d PT). The full fruit sampling occurred when the plants contained all red fruit (65 d PT) and terminal stage sampling as the plant appeared to wilt and began ending growth (67 d PT). At each assessment, four randomly selected plants from each treatment group (eight plants total) were dissected and the roots, stems, leaves, and fruit (if present) of each plant were sampled for the presence of $S$. enterica. Conventional plating and molecular procedures were used to analyze the plant tissues for $S$. enterica.

Stem and leaf analysis. Randomly selected leaves were aseptically removed from the plant using surface-sterilized scissors. To isolate only bacteria on the internal portion of the plant, all surfaces analyzed were sanitized with $70 \%$ ethanol (Klerks et al., 2007; Miles et al., 2009). The surface disinfection treatment was confirmed in preliminary work by surface-inoculating samples, allowing them to dry for $1 \mathrm{~h}$ under a flow hood, subjected to surface disinfection, and plating tissue homogenates (data not shown). One leaf from one branch from the top, middle, and lower portions of the tomato plant were included. The leaves were surface-sterilized by spraying with $70 \%$ ethanol solution and allowed to dry under a flow hood until no visible solution remained (Miles et al., 2009). The excised leaves were aseptically combined in one stomacher bag and treated as a single sample.

The stems were aseptically cut into $3-\mathrm{cm}$ portions beginning at 20,40 , and $60 \mathrm{~cm}$ (from the surface of the soil) and combined to form one sample (Miles et al., 2009). If the stem was not long enough to sample in intervals, the entire stem was sampled. The stem was surface-sterilized by spraying with $70 \%$ ethanol solution and allowed to dry under a flow hood until no visible ethanol remained (Miles et al., 2009). Stem pieces were then aseptically combined in one stomacher bag and treated as a single sample.

Ten milliliters of $0.1 \%$ buffered peptone water was added to each stomacher bag sample of either leaves or stems and stomached for $90 \mathrm{~s}$. Homogenates were streaked $(10 \mu \mathrm{L})$ onto XLT-4 agar. After $24 \mathrm{~h}$ incubation at $37{ }^{\circ} \mathrm{C}$, typical $S$. enterica colony formation was considered presumptive positives.

Root analysis. Roots were aseptically removed from the plant and washed in distilled water until no residual soil remained. The roots were surface-sterilized by spraying with $70 \%$ ethanol solution and allowed to dry under a laminar flow hood until no visible ethanol remained (Miles et al., 2009). The root sample was then combined with $10 \mathrm{~mL}$ of $0.1 \%$ buffered peptone water and stomached for $90 \mathrm{~s}$ in a sterile stomacher bag. The root homogenate was streaked $(10 \mu \mathrm{L})$ onto XLT-4 agar. After $24 \mathrm{~h}$ incubation at $37^{\circ} \mathrm{C}$, typical $S$. enterica colony formation was considered presumptive positives.
Tomato fruit analysis. Both green and red ripe tomato fruits were harvested from plants. Two fruit were randomly selected from each plant and sampled separately for $S$. enterica presence. Removed fruits were surface-sanitized by spraying $70 \%$ ethanol solution and then allowed to dry under a laminar flow hood until no visible ethanol remained (Miles et al., 2009). The fruit were then placed in individual sterile stomacher bags containing $10 \mathrm{~mL} 0.1 \%$ peptone water and stomached for $90 \mathrm{~s}$. The homogenate was streaked $(10 \mu \mathrm{L})$ onto XLT-4 agar. After $24 \mathrm{~h}$ incubation at $37{ }^{\circ} \mathrm{C}$, typical $S$. enterica colony formation was considered presumptive positives.

Enrichment procedures. The remaining homogenates from all root, stem, leaf, and fruit samples were enriched for S. enterica to identify cells that may not have been culturable from the initial sample. One-milliliter aliquots of each homogenate sample were separately inoculated into $10 \mathrm{~mL}$ Tetrathionate broth (Remel, Lenexa, KS). Tetrathionate broth was incubated at $37^{\circ} \mathrm{C}$ for $24 \mathrm{~h}$ and then streaked $(10 \mu \mathrm{L})$ onto XLT-4 agar. After $24 \mathrm{~h}$ incubation at $37{ }^{\circ} \mathrm{C}$, typical $S$. enterica colony formation was considered presumptive positives.

Polymerase chain reaction confirmation of $\mathrm{S}$. enterica in samples. After enrichment procedures, presumptive positive colonies from XLT-4 agar plates were confirmed to be $S$. enterica using polymerase chain reaction procedures (PCRs). Other bacteria can appear like $S$. enterica colonies on XLT-4 (red with black centers indicating $\mathrm{H}_{2} \mathrm{~S}$ production), resulting in the need for further confirmation. Each presumptive colony (one from each plate) was grown to $7 \mathrm{log}$ $\mathrm{CFU} \cdot \mathrm{mL}^{-1}$ in $10 \mathrm{~mL}$ TSB at $37^{\circ} \mathrm{C}$ for $24 \mathrm{~h}$ before PCR analysis. The culture was centrifuged at $7500 \mathrm{~g}$ for $10 \mathrm{~min}$ and then resuspended in sterile DI water. DNA was extracted using the Qiagen Generation Capture Plant Kit (Qiagen, Valencia, CA) per the manufacturer's instructions. PCR was performed using $100 \mathrm{ng}$ of purified DNA, $0.05 \mu \mathrm{M}$ invA (invA-112F 5' -TCGACAGAC GTAAGG 3' and invA-1920R 5'-GCGATA TTGGTG-3'), and $2 \times$ Taq Mastermix (New England Biolabs, Ipswich, MA). PCR conditions were as follows: initial denaturation at $96{ }^{\circ} \mathrm{C}$ for 2 min followed by 35 cycles of: $96{ }^{\circ} \mathrm{C}$ for $30 \mathrm{~s}, 55^{\circ} \mathrm{C}$ for $30 \mathrm{~s}$, and $72{ }^{\circ} \mathrm{C}$ for $1 \mathrm{~min}$ and a final extension of $10 \mathrm{~min}$ at $72^{\circ} \mathrm{C}$. All presumptive positive samples that were invA-negative were reamplified with a primer set for 16S rDNA that will amplify DNA from all bacteria. PCR products were visualized on a $1.5 \%$ agarose gel. Bands corresponding to a fragment size of 1800 confirmed amplification of the invA gene and confirmed the colony as $S$. enterica.

Statistical analysis. Each treatment group ( $S$. Newport-irrigated and control) contained 22 tomato plants (total of 44 plants) and four plants from each group were sampled at random at each of the five growth stages. Two tomato fruit from each plant were sampled separately. The correlation between the plant tissue type (root, stem, leaf, or fruit) 
and growth stage (14 d, early fruit, midfruit, full fruit, or terminal) on the presence of $S$. enterica was evaluated using $\chi^{2}$ analysis. Statistical analysis was completed using Statistical Analysis Software Version 9.1 (SAS Institute, Cary, NC).

\section{Results}

There were at total of 92 plant samples collected from each treatment group (20 stems, 20 leaves, 20 roots, and 32 fruit) over the course of the experiment. S. enterica was not recovered from plants irrigated with distilled water exclusively (control). The presence of $S$. enterica was detected consistently in the roots and stems of plants irrigated with the pathogen, but not consistently in the leaves or fruit (Table 1). Of the plants irrigated with $S$. Newport, 32\% (29 of 92) of samples before enrichment and 39\% (36 of 92) after enrichment were presumptively positive for $S$. enterica (Table 1). Of the 29 presumptive positive pre-enrichment samples, 20 were root samples, seven were stem samples, and two were leaf samples (Table 1). After enrichment procedures, an additional five stem and two tomato fruit samples were presumptively positive for $S$. enterica (Table 1).

Other bacteria isolated from plants can appear on XLT-4 agar as S. enterica (red with black centers indicating $\mathrm{H}_{2} \mathrm{~S}$ production); therefore, an additional step to confirm that the colonies were Salmonella was needed. All enriched homogenates were amplified for the invA gene (exclusive to $S$. enterica) using PCR (Table 1) (Rahn et al., 2002). Previous work in our laboratory established that the inv $A$ primers are specific for only members of S. enterica and do not amplify other members of the Enterobacteriaceae family that are likely to be associated with plant samples. After PCR, 13 of the 20 presumptive positive roots samples, eight of 12 stem samples, two of two leaf samples, and two of two fruit samples were confirmed as positive for $S$. enterica (Table 1). Therefore, $65 \%$ of roots, $40 \%$ of stems, $10 \%$ of leaves, and $6 \%$ of fruit were confirmed to be contaminated after

Table 1. Number of tomato plant samples [roots (R), stems (S), leaves (L), and fruit (F)] presumptively positive for S. enterica serotype Newport before enrichment of homogenate and postenrichment and confirmed using Salmonella invA-specific primers in plants irrigated with distilled water (control) $(\mathrm{n}=$ $4)$ and $S$. Newport $(\mathrm{n}=4)$ at different host growth stages grown under controlled environmental conditions. ${ }^{\mathrm{z}}$

\begin{tabular}{|c|c|c|c|c|c|c|c|c|c|c|c|c|}
\hline \multirow{2}{*}{$\begin{array}{l}\text { Sample } \\
\text { day }^{\mathrm{y}}\end{array}$} & \multicolumn{4}{|c|}{ Before enrichment } & \multicolumn{4}{|c|}{ Postenrichment } & \multicolumn{4}{|c|}{ Polymerase chain reaction } \\
\hline & $\overline{\mathrm{R}}$ & $\mathrm{S}$ & $\mathrm{L}$ & $\bar{F}$ & $\overline{\mathrm{R}}$ & $\mathrm{S}$ & $\mathrm{L}$ & $\mathrm{F}$ & $\overline{\mathrm{R}}$ & $\mathrm{S}$ & $\mathrm{L}$ & $\mathrm{F}$ \\
\hline$\overline{14}$ & 4 & 0 & 1 & $\mathrm{~N} / \mathrm{A}$ & 4 & 1 & 0 & $\mathrm{~N} / \mathrm{A}$ & 2 & 2 & 0 & $\mathrm{~N} / \mathrm{A}$ \\
\hline $49^{x}$ & 4 & 2 & 0 & 0 & 4 & 4 & 1 & 0 & 2 & 4 & 0 & 0 \\
\hline $56^{\mathrm{w}}$ & 4 & 3 & 1 & 0 & 4 & 3 & 1 & 1 & 2 & 1 & 1 & 1 \\
\hline $65^{\mathrm{v}}$ & 4 & 1 & 0 & 0 & 4 & 3 & 0 & 1 & 4 & 0 & 1 & 1 \\
\hline $67^{u}$ & 4 & 1 & 0 & 0 & 4 & 1 & 0 & 0 & 3 & 1 & 0 & 0 \\
\hline
\end{tabular}

${ }^{\mathrm{z}}$ No samples from control plants were positive for presence of Salmonella either pre- or post-enrichment. ${ }^{y}$ Sampling day was measured as the number of days after the seedlings were transplanted in accordance with plant maturity.

${ }^{\mathrm{x}} 49 \mathrm{~d}=$ early fruit, plants had two or more green fruit.

${ }^{w} 56 \mathrm{~d}=$ midfruit, plants contained a variety of red and green fruit.

${ }^{\mathrm{v}} 65 \mathrm{~d}=$ full fruit, plants contained all red fruit.

${ }^{\mathrm{u}} 67 \mathrm{~d}=$ terminal, plant began wilting and growth ended.

$\mathrm{N} / \mathrm{A}=$ not applicable. with 3 to $4 \log \mathrm{CFU} \cdot \mathrm{mL}^{-1}$ of a $S$. enterica cocktail when grown hydroponically in contaminated medium (Guo et al., 2002). Association of the pathogen with nonroot tissues in the mature tomato plant is less convincing. No significant evidence of $S$. enterica in stem, leaves, and tomato fruit samples was found after irrigating cherry gold and red round greenhouse tomato plants with $S$. Enteritidis (a nontomato outbreak-associated serovar) or $S$. Montevideo, respectively (Jablasone et al., 2004; Miles et al., 2009).

Serovars of $S$. enterica persist differently in tomato plant tissues (Guo et al., 2001). To date, no work has been completed with $S$. Newport (a predominant serovar associated with tomato-related foodborne illness). To our knowledge, there are no other studies evaluating irrigation water as a source for tomato plant contamination. This study was completed to help clarify whether application of $S$. Newport in the root zone could result in contamination of various tomato plant tissues when sampled throughout differing plant growth stages. There is evidence that $S$. Newport can internalize into the roots and leaves of other produce crops. When the potting medium of 33-d-old romaine lettuce plants was inoculated, roots and leaves were positive for $S$. Newport (Bernstein et al., 2007a). Leaves from 17- or 20-d-old plants were not contaminated, suggesting the plants' age or developmental stage may affect bacterial internalization (Bernstein et al., 2007a). Other $S$. enterica serovars have been detected in the aerial tissue of other plants (Franz et al., 2007; Jablasone et al., 2005; Klerks et al., 2007).

In this study, $S$. enterica was significantly less likely to be associated with leaves and fruit of tomatoes. Once aerial plant tissues such as leaves becoming contaminated, the pathogen encounters harsh physiochemical conditions (ultraviolet radiation, lack of available nutrients) that diminish survival rates (Doyle et al., 2008). S. enterica numbers on leaf tissue have been found to be 30- to 40-fold less than those detected on roots (Cooley et al., 2003).

Care was taken to inoculate the tomato plant at consistent intervals such that each contamination event would not specifically affect the presence or absence of contamination during sampling. The continuous inoculation of $S$. Newport in the root zone likely replenished the levels or encouraged persistence of the bacterium in soil surrounding the roots. Salmonella Newport is capable of persisting in potting medium for 4.7 to 10 weeks (Bernstein et al., 2007b). The presence of $S$. enterica inside plant tissues was evaluated by sanitizing the exterior of the sample before enumeration or enrichment. Plant samples were sanitized with $70 \%$ ethanol before sampling. This method was preliminarily examined in our laboratory before the study and found to be sufficient. Although ethanol removes $99.81 \%$ of $S$. enterica from the surface of leafy tissues (Klerks et al., 2007), it is likely that all bacteria were not been removed. In this study, with the exception of roots, $S$. enterica was not applied directly to 
the exterior of plant tissues (leaves, stems, or fruit); therefore, high levels of the pathogen on the exterior were unlikely. It is possible that more root samples were positive as a result of the high concentration of the $S$. Newport in the soil surrounding the root. A different surface sanitation method may have been more effective in sanitizing the exterior portions of the plant (Zhang et al., 2009).

Overall, $S$. Newport may be capable of contaminating aerial tomato plant tissues when consistently high levels are applied at the root site. $S$. Newport was able to be recovered from the plant at every growth stage, demonstrating the possibility for contaminated irrigation water to persist on tomato plant parts over the course of its existence. Contamination on the tomato fruit is of most significance. We found two tomatoes to be positive for $S$. enterica. Although this is significant, contamination was low because the fruit were only positive after enrichment. As a result of the use of a Biological Safety Level-2 pathogen, we were unable to conduct this study in a tomato field environment. Care was taken to control natural tomato-growing conditions as closely as possible, but these conditions are not representative of the complex natural systems found in agricultural fields. Persistent contamination of a plant would be highly unlikely in the agricultural field setting as a result of external factors (weather, ultraviolet exposure) and competition between $S$. enterica and other organisms in the phyllosphere. Ultimately, significant contamination of the edible tissue of the tomato plant (fruit) with $S$. Newport in field conditions is unlikely, even when consistently high levels of inoculum are applied to the root zone.

\section{Literature Cited}

Bernstein, N., S. Sela, and S. Neder-Lavon. 2007a. Assessment of contamination potential of let- tuce by Salmonella enterica serovar Newport added to the plant growing medium. J. Food Prot. 70:1717-1722.

Bernstein, N., S. Sela, and S. Neder-Lavon. 2007b. Effect of irrigation regimes on persistence of Salmonella enterica serovar Newport in small experimental pots designed for plant cultivation. Irrig. Sci. 26:1-8.

Centers for Disease Control and Prevention. 2008. Outbreak of Salmonella serotype Saintpaul infections associated with multiple raw produce items. MMWR 57:929-934.

Cooley, M.B., W.G. Miller, and R.E. Mandrell. 2003. Colonization of Arabidopsis thaliana with Salmonella enterica and enterohemorrhagic Escherichia coli $\mathrm{O} 157: \mathrm{H} 7$ and competition by Enterobacter asburiae. Appl. Environ. Microbiol. 69:4915-4926.

Dong, Y., A.L. Iniguez, and E.W. Triplett. 2003. Quantitative assessments of the host range and strain specificity of endophytic colonization by Klebsiella pneumoniae. Plant Soil 257:49-59.

Doyle, M.P. and M.C. Erickson. 2008. Summer meeting 2007-The problems with fresh produce: An overview. J. Appl. Microbiol. 105: 317-330.

Food and Drug Administration. 2007a. FDA implementing initiative to reduce tomato-related foodborne illnesses. 29 July 2009. <http://www.fda.gov/ NewsEvents/Newsroom/PressAnnouncements/ 2007/ucm108931.htm>.

Food and Drug Administration. 2007b. Tomato safety initiative. 29 July 2009. <http://www.fda. gov/Food/FoodSafety/Product-SpecificInformation/FruitsVegetablesJuices/FDAProduce SafetyActivities/ucm115334.htm>.

Franz, E., A.A. Visser, A.D. Van Diepeningen, M.M. Klerks, A.J. Termorshuizen, and A.H. van Bruggen. 2007. Quantification of contamination of lettuce by GFP-expressing Escherichia coli O157:H7 and Salmonella enterica serovar Typhimurium. Food Micro. 24:106-112.

Guo, X., J. Chen, R.E. Brackett, and L.R. Beuchat. 2001. Survival of Salmonellae on and in tomato plants from the time of inoculation at flowering and early stages of fruit development through fruit ripening. Appl. Environ. Microbiol. 67: $4760-4764$.

Guo, X., M.W. van Iersel, J. Chen, R.E. Brackett, and L.R. Beuchat. 2002. Evidence of associa- tion of Salmonellae with tomato plants grown hydroponically in inoculated nutrient solution. Appl. Environ. Microbiol. 68:3639-3643.

Hallman, J., A.A. Quadt-Hallman, W.F. Mahaffee, and J.W. Kloepper. 1997. Bacterial endophytes in agricultural crops. Can. Micro. 43: 895-914.

Jablasone, J., L.Y. Brovko, and M.W. Griffiths. 2004. A research note: The potential for transfer of Salmonella from irrigation water to tomatoes. J. Sci. Food Agr. 84:287-289.

Jablasone, J., K. Warriner, and M. Griffiths. 2005. Interactions of Escherichia coli O157:H7, Salmonella Typhimurium and Listeria monocytogenes plants cultivated in a gnotobiotic system. Int. J. Food Microbiol. 99:7-18.

Klerks, M.M., M. van Gent-Pelzer, E. Franz, C. Zijlstra, and A.H.C. van Bruggen. 2007. Physiological and molecular responses of lactuca sativa to colonization by Salmonella enterica serovar Dublin. Appl. Environ. Microbiol. 73: 4905-4914.

Miles, J.M., S.S. Sumner, R.R. Boyer, R.C. Williams, J.G. Latimer, and J.M. McKinney. 2009. Internalization of Salmonella enterica Montevideo into greenhouse tomato plants through contaminated irrigation water or seed stock. J. Food Prot. 72:849-852.

National Agricultural Statistics Service. 2009. Tomatoes, national statistics. 16 Dec. 2009. $<$ http://www.nass.usda.gov/QuickStats/index2. jsp $>$.

Rahn, K., S.A. De Grandis, R.C. Clarke, S.A. McEwen, J.E. Galan, C. Ginocchio, R. Curtiss, III, and C.L. Gyles. 2002. Amplification of an invA gene sequence of Salmonella typhimurium by polymerase chain reaction as a specific method of detection of Salmonella. Mol. Cell. Probes 6:271-279.

Shin, A. 2008. FDA officials narrow Salmonella warning to Mexican peppers. Washington Post. 13 Nov. 2009. <http://www.washingtonpost.com/ wp-dyn/content/article/2008/07/25/AR200807 2502226.html>.

Zhang, G., L. Ma, L.R. Beuchat, M.C. Erickson, V.H. Phelan, and M.P. Doyle. 2009. Evaluation of treatments for elimination of foodborne pathogens on the surface of leaves and roots of lettuce (Lactuca sativa L.). J. Food Prot. $72: 228-234$. 